\title{
MEAN GROWTH AND GEOMETRIC ZERO DISTRIBUTION OF SOLUTIONS OF LINEAR DIFFERENTIAL EQUATIONS
}

\author{
JANNE GRÖHN, ARTUR NICOLAU, AND JOUNI RÄTTYÄ
}

\begin{abstract}
The aim of this paper is to consider certain conditions on the coefficient $A$ of the differential equation $f^{\prime \prime}+A f=0$ in the unit disc, which place all normal solutions $f$ to the union of Hardy spaces or result in the zero-sequence of each non-trivial solution to be uniformly separated. The conditions on the coefficient are given in terms of Carleson measures.
\end{abstract}

\section{INTRODUCTION}

We consider solutions of the linear differential equation

$$
f^{\prime \prime}+A f=0
$$

in the unit disc $\mathbb{D}$ of the complex plane $\mathbb{C}$. Recall that, if $f_{1}$ and $f_{2}$ are linearly independent solutions of (1.1), then the Schwarzian derivative

$$
S_{w}=\left(\frac{w^{\prime \prime}}{w^{\prime}}\right)^{\prime}-\frac{1}{2}\left(\frac{w^{\prime \prime}}{w^{\prime}}\right)^{2}
$$

of the quotient $w=f_{1} / f_{2}$ satisfies $S_{w}=2 A$. One of our main objectives is to explore conditions on the coefficient $A$ placing all solutions of (1.1) to the union of Hardy spaces, while the other aim is to study the geometric zero distribution of non-trivial solutions $f \not \equiv 0$ of (1.1). In other words, we study restrictions of the Schwarzian derivative $S_{w}$ of a locally univalent meromorphic function $w=f_{1} / f_{2}$ that place $1 / w^{\prime}$ (which reduces to a constant multiple of $f_{2}^{2}$ ) to the union of Hardy spaces, and consider the geometric distribution of complex $a$-points of $w$ (which are precisely the zeros of the solution $f_{1}-a f_{2}$ if $a \in \mathbb{C}$, and the zeros of $f_{2}$ if $a=\infty$ ).

We begin by recalling some notation. For $0<p<\infty$, the Hardy space $H^{p}$ consists of those analytic functions in $\mathbb{D}$ for which

$$
\|f\|_{H^{p}}=\lim _{r \rightarrow 1^{-}}\left(\frac{1}{2 \pi} \int_{0}^{2 \pi}\left|f\left(r e^{i \theta}\right)\right|^{p} d \theta\right)^{1 / p}<\infty .
$$

A positive Borel measure $\mu$ on $\mathbb{D}$ is called a Carleson measure, if there exists a positive constant $C$ such that

$$
\int_{\mathbb{D}}|f(z)|^{p} d \mu(z) \leq C\|f\|_{H^{p}}^{p}, \quad f \in H^{p} .
$$

Date: November 14, 2021.

2010 Mathematics Subject Classification. Primary 34C10, 34M10.

Key words and phrases. Carleson measure; Hardy space; linear differential equation; oscillation theory; uniform separation.

The first author is supported by the Academy of Finland \#258125; the second author is supported in part by the grants MTM2011-24606 and 2014SGR 75; and the third author is supported in part by the Academy of Finland \#268009, by the Faculty of Science and Forestry of University of Eastern Finland \#930349, and by the grant MTM2011-26538. 
These measures were characterized by Carleson as those positive measures $\mu$ for which there exists a positive constant $K$ such that $\mu(Q) \leq K \ell(Q)$ for any Carleson square $Q \subset \mathbb{D}$, or equivalently, as those positive measures $\mu$ for which

$$
\sup _{a \in \mathbb{D}} \int_{\mathbb{D}} \frac{1-|a|^{2}}{|1-\bar{a} z|^{2}} d \mu(z)<\infty .
$$

For more information see [7, 9], for example.

The following spaces of analytic functions seem to be natural for the coefficient. For $0 \leq \alpha<\infty$, the growth space $H_{\alpha}^{\infty}$ contains those analytic functions $A$ in $\mathbb{D}$ for which

$$
\|A\|_{H_{\alpha}^{\infty}}=\sup _{z \in \mathbb{D}}\left(1-|z|^{2}\right)^{\alpha}|A(z)|<\infty
$$

For $0<p<\infty$, the analytic function $A$ in $\mathbb{D}$ is said to belong to the space $F^{p}$ if $|A(z)|^{p}\left(1-|z|^{2}\right)^{2 p-1} d m(z)$ is a Carleson measure, and we denote

$$
\|A\|_{F^{p}}=\left(\sup _{a \in \mathbb{D}} \int_{\mathbb{D}}|A(z)|^{p}\left(1-|z|^{2}\right)^{2 p-2}\left(1-\left|\varphi_{a}(z)\right|^{2}\right) d m(z)\right)^{1 / p} .
$$

Here $\varphi_{a}(z)=(a-z) /(1-\bar{a} z)$ and $d m(z)$ denotes the element of the Lebesgue area measure on $\mathbb{D}$. Note that $F^{p} \subsetneq H_{2}^{\infty}$ for any $0<p<\infty$ by subharmonicity. Correspondingly, the "little-oh" space $F_{0}^{p}$ (the closure of polynomials in $F^{p}$ ), which consists of those analytic functions in $\mathbb{D}$ for which $|A(z)|^{p}\left(1-|z|^{2}\right)^{2 p-1} d m(z)$ is a vanishing Carleson measure, and the space $H_{\alpha, 0}^{\infty}$, which contains those analytic functions in $\mathbb{D}$ for which

$$
\lim _{|z| \rightarrow 1^{-}}\left(1-|z|^{2}\right)^{\alpha}|A(z)|=0,
$$

satisfy the inclusion $F_{0}^{p} \subsetneq H_{2,0}^{\infty}$ for any $0<p<\infty$. It is known that:

(i) for each $0<p<\infty$ there exists a positive constant $\alpha=\alpha(p)$ such that, if $\|A\|_{F^{2}} \leq \alpha$, then all solutions $f$ of (1.1) belong to $H^{p}$, and each non-trivial solution $f$ has at most one zero in $\mathbb{D}$;

(ii) if $A \in F_{0}^{2}$, then all solutions $f$ of (1.1) belong to $\bigcap_{0<p<\infty} H^{p}$, and each non-trivial solution $f$ has at most finitely many zeros in $\mathbb{D}$.

The assertion (i) follows from [17, Theorem 1.7]; note that by choosing a sufficiently small $0<\alpha<\infty$ we have $\|A\|_{H_{2}^{\infty}} \leq 1$, and hence each solution $f$ of (1.1) vanishes at most once in $\mathbb{D}$ [14, Theorem 1]. The case (ii) is a consequence of [17, p. 789]; in this case $A \in H_{2,0}^{\infty}$, and hence all solutions have at most finitely many zeros in $\mathbb{D}$ [18, Theorem 1]. Notice also that each non-trivial solution of (1.1) has at most finitely many zeros provided that $A \in F^{2}$ is lacunary, since the lacunary series in $F^{2}$ and $F_{0}^{2}$ are same.

\section{Results}

Our results concern interrelationships of the properties

(i) all solutions of (1.1) belong to the union of Hardy spaces;

(ii) the zero-sequence of each non-trivial solution of (1.1) is uniformly separated;

(iii) the growth of the coefficient $A$.

In the light of the following results it seems plausible that $|A(z)|^{2}\left(1-|z|^{2}\right)^{3} d m(z)$ being a Carleson measure is sufficient for (i) and (ii). The following theorems give many partial results in this direction. For example, the existence of one nonvanishing solution allows us to reach this conclusion, see Corollary 3 . 
We begin with the geometric zero distribution of solutions of (1.1). Recall that the sequence $\left\{z_{n}\right\}_{n=1}^{\infty} \subset \mathbb{D}$ is called uniformly separated if

$$
\inf _{k \in \mathbb{N}} \prod_{n \in \mathbb{N} \backslash\{k\}}\left|\frac{z_{n}-z_{k}}{1-\bar{z}_{n} z_{k}}\right|>0,
$$

while $\left\{z_{n}\right\}_{n=1}^{\infty} \subset \mathbb{D}$ is said to be separated in the hyperbolic metric if there exists a constant $\delta=\delta\left(\left\{z_{n}\right\}_{n=1}^{\infty}\right)>0$ such that $\varrho_{p}\left(z_{n}, z_{k}\right)=\left|z_{n}-z_{k}\right| /\left|1-\bar{z}_{n} z_{k}\right|>\delta$ for all $n, k \in \mathbb{N}$ for which $n \neq k$.

Theorem 1. If $|A(z)|\left(1-|z|^{2}\right) d m(z)$ is a Carleson measure, then the zero-sequence of each non-trivial solution $f$ of (1.1) is uniformly separated.

Theorem 1 should be compared with [18, Theorem 3] which states that, if $A \in$ $H_{2}^{\infty}$, then the zero-sequence of each non-trivial solution $f$ of (1.1) is separated in the hyperbolic metric by a constant depending on $\|A\|_{H_{2}^{\infty}}$. For the interplay between the maximal growth of the coefficient $A$ and the minimal separation of the zeros of non-trivial solutions $f$ of (1.1), we refer to [4].

The proof of Theorem 1 relies on Theorem $\mathrm{A}$ below, according to which all solutions $f$ of (1.1) belong to the Nevanlinna class $N$, that is

$$
\sup _{0 \leq r<1} m(r, f)=\sup _{0 \leq r<1} \frac{1}{2 \pi} \int_{0}^{2 \pi} \log ^{+}\left|f\left(r e^{i \theta}\right)\right| d \theta<\infty
$$

if

$$
\int_{\mathbb{D}}|A(z)|\left(1-|z|^{2}\right) d m(z)<\infty
$$

Condition (2.2) is, of course, satisfied by the hypothesis of Theorem 1.

2.1. Differential equations with one non-vanishing solution. The following result based on [3, Corollary 7] introduces a factorization of solutions of (1.1), which does not have an apparent counterpart in general.

Theorem 2. Let $A$ be an analytic function in $\mathbb{D}$, and suppose that (1.1) admits a non-vanishing solution $g$.

(i) If $|A(z)|^{2}\left(1-|z|^{2}\right)^{3} d m(z)$ is a Carleson measure, then all non-trivial solutions $f$ of (1.1) can be factorized as $f=g W$, where $\log g \in$ BMOA, and either $\log W^{\prime} \in \mathrm{BMOA}$ (if $f$ is linearly independent to $g$ ) or $W$ is a non-zero complex constant (if $f$ is linearly dependent to $g$ ).

(ii) If $A \in H_{2}^{\infty}$, then all non-trivial solutions $f$ of (1.1) can be factorized as $f=g W$, where $\log g \in \mathcal{B}$, and either $\log W^{\prime} \in \mathcal{B}$ (if $f$ is linearly independent to $g$ ) or $W$ is a non-zero complex constant (if $f$ is linearly dependent to $g$ ).

Recall that BMOA consists those $f \in H^{2}$ for which $\left|f^{\prime}(z)\right|^{2}\left(1-|z|^{2}\right) d m(z)$ is a Carleson measure, and BMOA has the seminorm

$$
\|f\|_{\mathrm{BMOA}}=\left(\sup _{a \in \mathbb{D}} \int_{\mathbb{D}}\left|f^{\prime}(z)\right|^{2}\left(1-\left|\varphi_{a}(z)\right|^{2}\right) d m(z)<\infty\right)^{1 / 2} .
$$

The Bloch space $\mathcal{B}$ contains those analytic functions $f$ in $\mathbb{D}$ for which $f^{\prime} \in H_{1}^{\infty}$.

Corollary 3. Let $A$ be an analytic function in $\mathbb{D}$, and suppose that (1.1) admits a non-vanishing solution. If $|A(z)|^{2}\left(1-|z|^{2}\right)^{3} d m(z)$ is a Carleson measure, then all solutions of (1.1) belong to $H^{p}$ for some $0<p<\infty$, and the zero-sequence of each non-trivial solution $f$ of (1.1) is uniformly separated. 
The statement corresponding to Corollary 3 in the case of $A \in H_{2}^{\infty}$ is true without the assumption of existence of a non-vanishing solution: if $A \in H_{2}^{\infty}$, then all solutions of (1.1) belong to $H_{p}^{\infty}$ for some $p=p\left(\|A\|_{H_{2}^{\infty}}\right)>0$ [16, Example 1], and the zero-sequence of each non-trivial solution $f$ of (1.1) is separated in the hyperbolic metric by a positive constant depending on $\|A\|_{H_{2}^{\infty}}$ [18, Theorem 3].

The following result gives a complete description of the zero-free solutions in our setting.

Theorem 4. Let $A$ be an analytic function in $\mathbb{D}$.

(i) If $|A(z)|^{2}\left(1-|z|^{2}\right)^{3} d m(z)$ is a Carleson measure, then all non-vanishing solutions $f$ of (1.1) satisfy $\log f \in$ BMOA. Conversely, if (1.1) admits a zero-free solution $f$ satisfying $\log f \in \mathrm{BMOA}$, then $|A(z)|^{2}\left(1-|z|^{2}\right)^{3} d m(z)$ is a Carleson measure.

(ii) If $A \in H_{2}^{\infty}$, then all non-vanishing solutions $f$ of (1.1) satisfy $\log f \in \mathcal{B}$. Conversely, if (1.1) admits a zero-free solution $f$ satisfying $\log f \in \mathcal{B}$, then $A \in H_{2}^{\infty}$.

In the case of Theorem 4(i) all non-vanishing solutions $f$ of (1.1) are in fact outer functions in Hardy spaces, see [7, Corollary 3, p. 34]. Note that this property restricts not only the growth of non-vanishing solutions but also the rate at which they may decay to zero. Correspondingly, if $f$ is a zero-free solution of (1.1) with $A \in H_{2}^{\infty}$, then Theorem 4 (ii) implies that there exists a constant $p=p\left(\|A\|_{H_{2}^{\infty}}\right)$ with $0<p<\infty$ such that $(1-|z|)^{p} \lesssim|f(z)| \lesssim(1-|z|)^{-p}$ for all $z \in \mathbb{D}$. We employ the notation $a \asymp b$, which is equivalent to the conditions $a \lesssim b$ and $b \lesssim a$, where the former means that there exists a constant $C>0$ such that $a \leq C b$, and the latter is defined analogously.

For example, the argument above asserts that the singular inner function

$$
f(z)=\exp (-(1+z) /(1-z)), \quad z \in \mathbb{D},
$$

cannot be a solution of (1.1) with $A \in H_{2}^{\infty}$. This is also easily verified by a direct computation, since in this case $A(z)=-4 z(1-z)^{-4}$ by (1.1). In particular, the boundedness of one solution of (1.1) is not sufficient to guarantee that $A \in H_{2}^{\infty}$.

One of the main tools concerning the results in Section [2.1 is [ [3, Corollary 7], which also induces a growth estimate for the non-vanishing solutions of (1.1).

Proposition 5. Let $A$ be analytic in $\mathbb{D}$. If $f$ is a non-vanishing solution of (1.1), then

$$
\frac{1}{2 \pi} \int_{0}^{2 \pi}\left|\log \frac{f\left(r e^{i \theta}\right)}{f(0)}\right|^{2} d \theta \lesssim r^{2}\left|\frac{f^{\prime}(0)}{f(0)}\right|^{2}+r^{2} \int_{D(0, r)}|A(z)|^{2}\left(1-|z|^{2}\right)^{3} d m(z)
$$

for all $0<r<1$.

By Proposition 5] and [7, Corollary 3, p. 34] all non-vanishing solutions of (1.1) are outer functions in the Nevanlinna class provided that

$$
\int_{\mathbb{D}}|A(z)|^{2}\left(1-|z|^{2}\right)^{3} d m(z)<\infty .
$$

It would be desirable to show that (2.3) (we may also assume that $A \in H_{2}^{\infty}$ ) is sufficient to place all solutions of (1.1) in $N$. This would improve the results in the 
literature (under the additional condition $A \in H_{2}^{\infty}$ ), since

$$
\begin{aligned}
\int_{\mathbb{D}}|A(z)|^{2}\left(1-|z|^{2}\right)^{3} d m(z) & \leq\|A\|_{H_{2}^{\infty}} \int_{\mathbb{D}}|A(z)|\left(1-|z|^{2}\right) d m(z) \\
& \leq\|A\|_{H_{2}^{\infty}}^{3 / 2} \int_{\mathbb{D}}|A(z)|^{1 / 2} d m(z) .
\end{aligned}
$$

The last integral in (2.4) appears in the growth estimate [16, Theorem 5], which is obtained by Herold's comparison theorem, while the intermediate integral shows up in Theorem A, which is proved by integrating (1.1) and applying the Gronwall lemma.

We close our discussion on non-vanishing solutions by the following result, which shows that there are differential equations (1.1) in $\mathbb{D}$ having no zero-free solutions. The proof of Theorem [6 was constructed jointly with Professor O. Roth.

Theorem 6. There exists a locally univalent meromorphic function in $\mathbb{D}$, which maps $\mathbb{D}$ onto the extended complex plane.

2.2. Normal solutions of differential equations. By Corollary 3 , the existence of one non-vanishing solution of (1.1) allows us to survey many specific properties of all solutions of (1.1). We now drop the additional assumption on the existence of a non-vanishing solution, and proceed to study solutions which may have zeros, but whose behavior around their zeros is in a certain sense regular. This leads us to the concept of normality. Recall that the meromorphic function $f$ in $\mathbb{D}$ is called normal (in the sense of Lehto and Virtanen) if and only if

$$
\sigma(f)=\sup _{z \in \mathbb{D}}\left(1-|z|^{2}\right) \frac{\left|f^{\prime}(z)\right|}{1+|f(z)|^{2}}<\infty
$$

for more information on normal functions, see for example [13].

Proposition 7. Let $f$ be a non-trivial solution of (1.1) with $A \in H_{2}^{\infty}$, and let $\left\{z_{n}\right\}_{n=1}^{\infty}$ be the zero-sequence of $f$. Then, the following conditions are equivalent:

(i) $f$ is normal;

(ii) $\sup _{n \in \mathbb{N}}\left(1-\left|z_{n}\right|^{2}\right)\left|f^{\prime}\left(z_{n}\right)\right|<\infty$;

(iii) $f$ is uniformly bounded in $\bigcup_{n=1}^{\infty} D\left(z_{n}, c\left(1-\left|z_{n}\right|\right)\right)$ for some $0<c<1$.

By Proposition 7 every solution of (1.1) with $A \in H_{2}^{\infty}$, which has only finitely many zeros, is normal. In particular, if $A \in H_{2,0}^{\infty}$, then all solutions of (1.1) are normal [18, Theorem 1] — yet all non-trivial solutions may lie outside $N$ [11, pp. 57-58].

Corollary 9 below states that all normal solutions of (1.1) belong to certain Hardy space, under the assumption that $|A(z)|^{2}\left(1-|z|^{2}\right)^{3} d m(z)$ is a Carleson measure. The proof of Corollary 9 is based on the following result, whose proof bears similarity to that of [3, pp. 105-107].

Theorem 8. If $w$ is a meromorphic function in $\mathbb{D}$ such that $\left|S_{w}(z)\right|^{2}\left(1-|z|^{2}\right)^{3} d m(z)$ is a Carleson measure and $w^{\prime}$ is normal, then for all sufficiently small $0<p<$ $\infty$ there exists a constant $C=C\left(p,\left\|S_{w}\right\|_{F^{2}}, \sigma\left(w^{\prime}\right)\right)$ with $1<C<\infty$ such that $\left\|1 / w^{\prime}\right\|_{H^{p}} \leq C$.

If we assume in Theorem 8 that $w$ has only finitely many poles (which all are simple, since the analyticity of $S_{w}$ implies that $w$ is locally univalent), then the assumption on the normality of $w^{\prime}$ is not needed. In fact, this follows from the 
other assumptions, since in this case $f=1 / \sqrt{w^{\prime}}$ is a solution of $f^{\prime \prime}+(1 / 2) S_{w} f=0$ having only finitely many zeros, and hence $f$ is normal by Proposition 7 . As a consequence we deduce that $w^{\prime}$ is normal.

Corollary 9. If $|A(z)|^{2}\left(1-|z|^{2}\right)^{3} d m(z)$ is a Carleson measure, then all normal solutions $f$ of (1.1) belong to $H^{p}$ for any sufficiently small $0<p<\infty$.

\section{Proof of Theorem 1}

The following result concerns the growth of solutions of linear differential equations. Recall that $\log ^{+} x=\max \{\log x, 0\}$ and $m(r, g)$ is the Nevanlinna proximity function of $g$, as in (2.1).

Theorem A ([10, Theorem 4.5]). If

$$
\int_{\mathbb{D}}|B(\zeta)|\left(1-|\zeta|^{2}\right) d m(\zeta)<\infty
$$

then every solution $g$ of $g^{\prime \prime}+B g=0$ is of bounded characteristic, and

$$
m(r, g) \leq \log ^{+}\left(|g(0)|+\left|g^{\prime}(0)\right|\right)+K \int_{D(0, r)}|B(\zeta)|\left(1-|\zeta|^{2}\right) d m(\zeta), \quad 0 \leq r<1,
$$

where $0<K<\infty$ is an absolute constant.

We proceed to prove Theorem 1 , Let $\kappa \in \mathbb{D}$. If $f$ is a solution of (1.1), then

$$
g_{\kappa}(\zeta)=\gamma f\left(\varphi_{\kappa}(\zeta)\right)\left(\varphi_{\kappa}^{\prime}(\zeta)\right)^{-1 / 2}, \quad \gamma \in \mathbb{C}
$$

is a solution of

$$
g_{\kappa}^{\prime \prime}+B_{\kappa} g_{\kappa}=0, \quad B_{\kappa}(\zeta)=A\left(\varphi_{\kappa}(\zeta)\right) \varphi_{\kappa}^{\prime}(\zeta)^{2}+\frac{1}{2} S_{\varphi_{\kappa}}(\zeta), \quad \zeta \in \mathbb{D}
$$

see for example [12, p. 394] or [16, Lemma 1]. Here $\varphi_{\kappa}(\zeta)=(\kappa-\zeta) /(1-\bar{\kappa} \zeta)$, and hence the Schwarzian derivative $S_{\varphi_{\kappa}}$ vanishes identically. The change of variable $z=\varphi_{\kappa}(\zeta)$ implies

$$
\sup _{\kappa \in \mathbb{D}} \int_{\mathbb{D}}\left|B_{\kappa}(\zeta)\right|\left(1-|\zeta|^{2}\right) d m(\zeta)=\sup _{\kappa \in \mathbb{D}} \int_{\mathbb{D}}|A(z)|\left(1-\left|\varphi_{\kappa}(z)\right|^{2}\right) d m(z)=\|A\|_{F^{1}},
$$

and by means of Theorem $\mathrm{A}$ we obtain

$$
m\left(r, g_{\kappa}\right) \leq \log ^{+}\left(\left|g_{\kappa}(0)\right|+\left|g_{\kappa}^{\prime}(0)\right|\right)+K\|A\|_{F^{1}}, \quad 0 \leq r<1, \quad \kappa \in \mathbb{D},
$$

for some absolute constant $0<K<\infty$.

Let $\left\{z_{n}\right\}_{n=1}^{\infty}$ be the zero-sequence of $f$. For each $k \in \mathbb{N}$, we have $g_{z_{k}}(0)=0$, and this zero of $g_{z_{k}}$ at the origin is simple as all the zeros of all non-trivial solutions of (3.1) are. Since $g_{z_{k}}^{\prime}(0) \neq 0$, we may normalize the solution $g_{z_{k}}$ to satisfy

$$
g_{z_{k}}^{\prime}(0)=\gamma f^{\prime}\left(z_{k}\right)\left(\left|z_{k}\right|^{2}-1\right)^{1 / 2}=1
$$

by choosing the constant $\gamma=\gamma(f, k)$ appropriately. We proceed to prove that the Blaschke sum regarding the zeros of the normalized solution $g_{z_{k}}$ of (3.1) is uniformly bounded for all $k \in \mathbb{N}$. Without loss of generality, we may suppose that the zeros $\left\{\zeta_{n, k}\right\}_{n=1}^{\infty}$ of $g_{z_{k}}$ satisfy $\left|\zeta_{1, k}\right|=0<\left|\zeta_{2, k}\right| \leq\left|\zeta_{3, k}\right| \leq \cdots$. By applying Jensen's formula to $z \mapsto z^{-1} g_{z_{k}}(z)$ results in

$$
\frac{1}{2 \pi} \int_{0}^{2 \pi} \log \left|g_{z_{k}}\left(r e^{i \theta}\right)\right| d \theta=\sum_{\substack{n \in \mathbb{N} \\ 0<\left|\zeta_{n, k}\right|<r}} \log \frac{r}{\left|\zeta_{n, k}\right|}+\log r, \quad 0<r<1
$$


Letting $r \rightarrow 1^{-}$, and taking account on (3.2), we get

$$
\sup _{k \in \mathbb{N}} \sum_{n=2}^{\infty}\left(1-\left|\zeta_{n, k}\right|\right) \leq K\|A\|_{F^{1}}
$$

Since the zeros of $g_{z_{k}}$ are precisely the images of the zeros of $f$ under the mapping $\varphi_{z_{k}}$, (3.3) implies that the zero-sequence $\left\{z_{n}\right\}_{n=1}^{\infty}$ of $f$ satisfies

$$
\sup _{k \in \mathbb{N}} \sum_{n \neq k}\left(1-\left|\frac{z_{n}-z_{k}}{1-\bar{z}_{n} z_{k}}\right|\right)=\sup _{k \in \mathbb{N}} \sum_{n=2}^{\infty}\left(1-\left|\zeta_{n, k}\right|\right) \leq K\|A\|_{F^{1}} .
$$

Since $A \in H_{2}^{\infty},\left\{z_{n}\right\}_{n=1}^{\infty}$ is separated [18, Theorem 3]. Let $0<\delta<1$ be a constant such that $\varrho_{p}\left(z_{n}, z_{k}\right) \geq \delta$ for all natural numbers $n \neq k$, where $\varrho_{p}$ stands for the pseudo-hyperbolic distance. Now (3.4), and the inequality $-\log x \leq x^{-1}(1-x)$ for $0<x<1$, imply

$$
\sup _{k \in \mathbb{N}} \sum_{n \neq k}-\log \left|\frac{z_{n}-z_{k}}{1-\bar{z}_{n} z_{k}}\right| \leq \frac{1}{\delta} \sup _{k \in \mathbb{N}} \sum_{n \neq k}\left(1-\left|\frac{z_{n}-z_{k}}{1-\bar{z}_{n} z_{k}}\right|\right) \leq \frac{K\|A\|_{F^{1}}}{\delta} .
$$

We conclude that $\left\{z_{n}\right\}_{n=1}^{\infty}$ is uniformly separated.

\section{Proof of Theorem 2}

(i) Let $\left\{f_{1}, f_{2}\right\}$ be a solution base of (1.1) such that $g=f_{2}$ is non-vanishing, and the Wronskian determinant $W\left(f_{1}, f_{2}\right)=-1$. Then $w=f_{1} / f_{2}$ is analytic, locally univalent, and it satisfies $S_{w}=2 A$ and $w^{\prime}=f_{2}^{-2}$. Define $h_{\zeta}(z)=\log w^{\prime}\left(\varphi_{\zeta}(z)\right)$, where $\varphi_{\zeta}(z)=(\zeta-z) /(1-\bar{\zeta} z)$ and $\zeta \in \mathbb{D}$. According to [3, Corollary 7 ,

$$
\left\|h_{\zeta}-h_{\zeta}(0)\right\|_{H^{2}}^{2} \lesssim\left|h_{\zeta}^{\prime}(0)\right|^{2}+\int_{\mathbb{D}}\left|h_{\zeta}^{\prime \prime}(z)-h_{\zeta}^{\prime}(z)^{2} / 2\right|^{2}\left(1-|z|^{2}\right)^{3} d m(z) .
$$

By a direct computation,

$h_{\zeta}^{\prime}(z)=\frac{w^{\prime \prime}\left(\varphi_{\zeta}(z)\right) \varphi_{\zeta}^{\prime}(z)}{w^{\prime}\left(\varphi_{\zeta}(z)\right)}, \quad h_{\zeta}^{\prime \prime}(z)-\frac{h_{\zeta}^{\prime}(z)^{2}}{2}=S_{w}\left(\varphi_{\zeta}(z)\right)\left(\varphi_{\zeta}^{\prime}(z)\right)^{2}+\frac{w^{\prime \prime}\left(\varphi_{\zeta}(z)\right) \varphi_{\zeta}^{\prime \prime}(z)}{w^{\prime}\left(\varphi_{\zeta}(z)\right)}$

which implies

$$
\begin{aligned}
\sup _{\zeta \in \mathbb{D}}\left\|h_{\zeta}-h_{\zeta}(0)\right\|_{H^{2}}^{2} \lesssim & \left\|w^{\prime \prime} / w^{\prime}\right\|_{H_{1}^{\infty}}^{2} \\
& +\sup _{\zeta \in \mathbb{D}} \int_{\mathbb{D}}\left|S_{w}\left(\varphi_{\zeta}(z)\right)\right|^{2}\left|\varphi_{\zeta}^{\prime}(z)\right|^{4}\left(1-|z|^{2}\right)^{3} d m(z) \\
& +\sup _{\zeta \in \mathbb{D}} \int_{\mathbb{D}}\left|\frac{w^{\prime \prime}\left(\varphi_{\zeta}(z)\right)}{w^{\prime}\left(\varphi_{\zeta}(z)\right)}\right|^{2}\left|\varphi_{\zeta}^{\prime \prime}(z)\right|^{2}\left(1-|z|^{2}\right)^{3} d m(z) .
\end{aligned}
$$

The right-hand side of (4.1) is finite, since $S_{w} \in H_{2}^{\infty}$ [19, Theorem 2], while (4.2) reduces to $\left\|S_{w}\right\|_{F^{2}}^{2}$. Furthermore, (4.3) is finite, since it is bounded by

$$
\left\|w^{\prime \prime} / w^{\prime}\right\|_{H_{1}^{\infty}}^{2} \sup _{\zeta \in \mathbb{D}} \int_{\mathbb{D}}\left|\frac{\varphi_{\zeta}^{\prime \prime}(z)}{\varphi_{\zeta}^{\prime}(z)}\right|^{2}\left(1-|z|^{2}\right) d m(z)<\infty .
$$

We conclude that $\log w^{\prime}$ belongs to BMOA.

Now, any solution $f \not \equiv 0$ of (1.1), which is linearly independent to $f_{2}$, can be written as $f=\alpha f_{1}+\beta f_{2}=(\alpha w+\beta) f_{2}$, where $\alpha \neq 0$. Then, functions $W=\alpha w+\beta$ and $g=f_{2}$ satisfy the assertion, since $\log W^{\prime}=\log \alpha+\log w^{\prime} \in$ BMOA and $\log g=-2 \log w^{\prime} \in \mathrm{BMOA}$ by the argument above. Moreover, all solutions $f$ of 
(1.1), which are linearly dependent to $f_{2}$, satisfy $\log f=\log \beta+\log f_{2} \in$ BMOA. The assertion (i) is proved.

(ii) Let $\left\{f_{1}, f_{2}\right\}$ be a solution base of (1.1) such that $g=f_{2}$ is non-vanishing, and the Wronskian determinant $W\left(f_{1}, f_{2}\right)=-1$. Then $w=f_{1} / f_{2}$ is analytic, locally univalent, and it satisfies $S_{w}=2 A$ and $w^{\prime}=f_{2}^{-2}$. By the assumption $S_{w} \in H_{2}^{\infty}$, which implies $\log w^{\prime} \in \mathcal{B}$ [19, Theorem 2]. The assertion of (ii) follows as above.

\section{Proof of Corollary 3}

The following auxiliary result, which is well-known by experts, is proved for the convenience of the reader.

Lemma 10. If $W$ is a locally univalent analytic function in $\mathbb{D}$ such that $\log W^{\prime} \in$ BMOA, then all finite a-points of $W$ (i.e. solutions of $W(z)=a \in \mathbb{C}$ ) are uniformly separated.

Proof. It suffices to prove the assertion for the zeros, for otherwise we may consider the zeros of $W(z)-a$ for $a \in \mathbb{C}$. Let $\left\{z_{n}\right\}_{n=1}^{\infty}$ be the zero-sequence of $W$, and define

$$
h_{z_{n}}(z)=-\frac{W\left(\varphi_{z_{n}}(z)\right)}{W^{\prime}\left(z_{n}\right)\left(1-\left|z_{n}\right|^{2}\right)}, \quad z \in \mathbb{D}, \quad n \in \mathbb{N} .
$$

Then $h_{z_{n}}(0)=0$ and $h_{z_{n}}^{\prime}(0)=1$. Now $\log h_{z_{n}}^{\prime}$ is well-defined and analytic, as $W$ is locally univalent. By the Littlewood-Paley identity [9, Lemma 3.1, p. 228],

$$
\begin{aligned}
\left\|\log h_{z_{n}}^{\prime}\right\|_{\mathrm{BMOA}}^{2} \asymp \sup _{a \in \mathbb{D}} \int_{\mathbb{D}}\left|\frac{W^{\prime \prime}\left(\varphi_{z_{n}}(z)\right)}{W^{\prime}\left(\varphi_{z_{n}}(z)\right)} \varphi_{z_{n}}^{\prime}(z)+\frac{\varphi_{z_{n}}^{\prime \prime}(z)}{\varphi_{z_{n}}^{\prime}(z)}\right|^{2}\left(1-\left|\varphi_{a}(z)\right|^{2}\right) d m(z) \\
\lesssim \sup _{a \in \mathbb{D}} \int_{\mathbb{D}}\left|\frac{W^{\prime \prime}(\zeta)}{W^{\prime}(\zeta)}\right|^{2}\left(1-\left|\varphi_{a}\left(\varphi_{z_{n}}(\zeta)\right)\right|^{2}\right) d m(\zeta) \\
\quad+\sup _{a \in \mathbb{D}} \int_{\mathbb{D}}\left|\frac{\varphi_{z_{n}}^{\prime \prime}(z)}{\varphi_{z_{n}}^{\prime}(z)}\right|^{2}\left(1-\left|\varphi_{a}(z)\right|^{2}\right) d m(z) \\
\lesssim\left\|\log W^{\prime}\right\|_{\mathrm{BMOA}}^{2}+1, \quad n \in \mathbb{N} .
\end{aligned}
$$

According to [5, Theorem 1] we have $\sup _{n \in \mathbb{N}}\left\|h_{z_{n}}^{\prime}\right\|_{H^{p}} \lesssim 1$ for sufficiently small $p=p\left(\left\|\log W^{\prime}\right\|_{\text {BMOA }}\right)$ with $0<p<\infty$, and hence $\sup _{n \in \mathbb{N}}\left\|h_{z_{n}}\right\|_{H^{p}} \lesssim 1$ by [7, Theorem 5.12]. We deduce, by means of $\log x \leq p^{-1} x^{p}$ for $0<x<\infty$, that

$$
\sup _{n \in \mathbb{N}} \frac{1}{2 \pi} \int_{0}^{2 \pi} \log \left|h_{z_{n}}\left(r e^{i \theta}\right)\right| d \theta \lesssim \sup _{n \in \mathbb{N}}\left\|h_{z_{n}}\right\|_{H^{p}}^{p} \lesssim 1
$$

By applying Jensen formula to $z \mapsto z^{-1} h_{z_{n}}(z)$, and arguing as in the proof of Theorem 1, we obtain the assertion.

We proceed to prove Corollary 3. Suppose that (1.1) admits a non-vanishing solution $g$, and let $|A(z)|^{2}\left(1-|z|^{2}\right)^{3} d m(z)$ be a Carleson measure. By Theorem 2(i) every non-trivial solution $f$ of (1.1) can be represented as $f=g W$, where $\log g \in$ BMOA, and either $W$ is locally univalent such that $\log W^{\prime} \in \mathrm{BMOA}$ or $W \in \mathbb{C} \backslash\{0\}$.

If $\log W^{\prime} \in \mathrm{BMOA}$, then $W^{\prime}=\exp \left(\log W^{\prime}\right)$ belongs to some Hardy space by $[$, Theorem 1]. This implies that also $W$ is in some Hardy space [7, Theorem 5.12]. Analogously, $\log g \in$ BMOA implies that $g=\exp (\log g)$ belongs to some Hardy space. In conclusion, under the assumptions of Corollary 3 every solution of (1.1) can be represented as a product of two Hardy functions and hence every solution of (1.1) belongs to certain (fixed) Hardy space. 
Every non-trivial solution of (1.1), which is linearly dependent to $g$, is zerofree, while the zero-sequence of every non-trivial solution of (1.1), which is linearly independent to $g$, is uniformly separated by Lemma 10.

\section{Proof of Theorem 4}

(i) The first part of the assertion follows directly from the proof of Theorem 2 (i), since $g=f_{2}$ can be chosen to be any non-vanishing solution of (1.1).

Conversely, suppose that (1.1) possesses a zero-free solution $f$ such that $\log f \in$ BMOA. Let $\{f, g\}$ be a solution base of (1.1) such that $W(f, g)=1$, and let $w=g / f$. Now $w^{\prime}$ is locally univalent analytic function such that $\log w^{\prime}=\log 1 / f^{2} \in$ BMOA. Moreover, the Schwarzian derivative $S_{w}=2 A$ is analytic in $\mathbb{D}$. Since

$$
\left|S_{w}(z)\right|^{2} \lesssim\left|\left(\frac{w^{\prime \prime}}{w^{\prime}}\right)^{\prime}(z)\right|^{2}+\left|\frac{w^{\prime \prime}(z)}{w^{\prime}(z)}\right|^{4}, \quad z \in \mathbb{D},
$$

and $\log w^{\prime} \in \mathrm{BMOA} \subset \mathcal{B}$, standard estimates show that $\left|S_{w}(z)\right|^{2}\left(1-|z|^{2}\right)^{3} d m(z)$ is a Carleson measure. The assertion of (i) follows.

(ii) Let $f$ be a zero-free solution of (1.1) with $A \in H_{2}^{\infty}$, and let $\{f, g\}$ be a solution base such that $W(f, g)=1$. Define $w=g / f$. Then $w$ is a locally univalent analytic function satisfying $S_{w}=2 A \in H_{2}^{\infty}$. By [19, Theorem 2] the pre-Schwarzian derivative $w^{\prime \prime} / w^{\prime}=\left(\log w^{\prime}\right)^{\prime} \in H_{1}^{\infty}$, and hence $\log f=-2^{-1} \log w^{\prime} \in \mathcal{B}$.

Conversely, suppose that (1.1) possesses a zero-free solution $f$ such that $\log f \in \mathcal{B}$. Now, $A=-f^{\prime \prime} / f=-\left(f^{\prime} / f\right)^{\prime}-\left(f^{\prime} / f\right)^{2} \in H_{2}^{\infty}$, which concludes the proof of Theorem 4 ,

\section{Proof of Proposition 5}

Let $f$ be a non-vanishing solution of (1.1), and let $\{f, g\}$ be a solution base of (1.1) such that $W(f, g)=1$. Define $w=g / f$, and notice that $w^{\prime}$ is a locally univalent analytic function such that $w^{\prime}=1 / f^{2}$. An application of [3, Corollary 7] with $\varphi(z)=\log \left(w^{\prime}(r z) r\right)$ yields

$$
\frac{1}{2 \pi} \int_{0}^{2 \pi}\left|\log \frac{w^{\prime}\left(r e^{i \theta}\right)}{w^{\prime}(0)}\right|^{2} d \theta \lesssim r^{2}\left|\frac{w^{\prime \prime}(0)}{w^{\prime}(0)}\right|^{2}+r^{2} \int_{\mathbb{D}}\left|S_{w}(r z)\right|^{2}\left(1-|z|^{2}\right)^{3} r^{2} d m(z)
$$

for $0<r<1$. This implies

$$
\frac{4}{2 \pi} \int_{0}^{2 \pi}\left|\log \frac{f\left(r e^{i \theta}\right)}{f(0)}\right|^{2} d \theta \lesssim 4 r^{2}\left|\frac{f^{\prime}(0)}{f(0)}\right|^{2}+r^{2} \int_{D(0, r)}\left|S_{w}(\zeta)\right|^{2}\left(1-\frac{|\zeta|^{2}}{r^{2}}\right)^{3} d m(\zeta)
$$

for $0<r<1$, which proves the assertion.

\section{Proof of Theorem 6}

Let $\widehat{\mathbb{C}}=\mathbb{C} \cup\{\infty\}$ denote the extended complex plane. Define $R: \widehat{\mathbb{C}} \rightarrow \widehat{\mathbb{C}}$ by $R(z)=z+1 /\left(2 z^{2}\right)$. Then $R$ is a rational function of degree three, and $R$ is locally univalent in $\Omega=\widehat{\mathbb{C}} \backslash\left\{\zeta_{1}, \zeta_{2}, \zeta_{3}, 0\right\}$, where $\zeta_{1}, \zeta_{2}$ and $\zeta_{3}$ are the three cube roots of unity.

Note that $R(z)=w$ has a solution $z \in \Omega$ for any $w \in \widehat{\mathbb{C}}$. First, the function $R$ takes the value $w=\infty$ at the point $z=\infty \in \Omega$. Second, if $w \in \mathbb{C}$, then $z=0$ cannot be a solution of $R(z)=w$. Hence $R(z)=w$ is equivalent to $2 z^{3}-2 w z^{2}+1=0$, which has a solution $z \in \Omega$ for any $w \in \mathbb{C}$. 
Let $M$ be the inversion $M(z)=1 / z$, and let $M^{-1}(\Omega)$ be the pre-image set of $\Omega$. Now, define $\Pi: \mathbb{D} \rightarrow M^{-1}(\Omega)$ to be a universal covering map. Since $M^{-1}(\Omega)$ is a plane set whose complement in $\mathbb{C}$ contains three points, we may assume that $\Pi$ is analytic [6, p. 125, Theorem 5.1]. The asserted function is $R \circ M \circ \Pi: \mathbb{D} \rightarrow \widehat{\mathbb{C}}$. This composition is locally univalent, since each function itself is locally univalent. The composition is surjective by the construction.

\section{Auxiliary results for Proposition 7 and Theorem 8}

If $w$ is meromorphic in $\mathbb{D}$ and $S_{w} \in H_{2}^{\infty}$, then $w \in \mathcal{U}(\eta)$ for some sufficiently small $\eta=\eta\left(\left\|S_{w}\right\|_{H_{2}^{\infty}}\right)$ by Nehari's theorem [16, Corollary 6.4]. Here $w \in \mathcal{U}(\eta)$ means that $w$ is meromorphic and uniformly locally univalent in $\mathbb{D}$, or equivalently, there exists $0<\eta \leq 1$ such that $w$ is meromorphic and univalent in each pseudo-hyperbolic $\operatorname{disc} \Delta_{p}(a, \eta)$ for $a \in \mathbb{D}$.

Denote by $\mathcal{P}_{w}$ the (discrete) set of poles of the meromorphic function $w$ in $\mathbb{D}$. Let $w \in \mathcal{U}(\eta)$ for some $0<\eta \leq 1$, and let $w^{\prime}$ be a normal function such that $\mathcal{P}_{w} \neq \emptyset$. By the Lipschitz-continuity of normal functions (as mappings from $\mathbb{D}$ equipped with the hyperbolic metric to the Riemann sphere with the chordal metric), there exists a constant $s=s\left(\sigma\left(w^{\prime}\right)\right)$ with $0<s<1$ such that

$$
\left|w^{\prime}(z)\right| \geq 1, \quad z \in \Delta_{p}(a, s), \quad a \in \mathcal{P}_{w} ;
$$

see for example [20, Theorem 1].

The following lemma lists some elementary properties of uniformly locally univalent functions, which are needed later. It is a local version of the well-known result according to which $\log w^{\prime} \in \mathcal{B}$ for all analytic and univalent functions $w$ in $\mathbb{D}$.

Lemma 11. Let $w \in \mathcal{U}(\eta)$ for some $0<\eta \leq 1$, and let $0<s<1$ be fixed. Suppose that $a \in \mathbb{D}$ is any point satisfying $\varrho_{p}\left(a, \mathcal{P}_{w}\right) \geq s$.

(i) Then

$$
\left|\frac{w^{\prime \prime}(a)}{w^{\prime}(a)}\right|\left(1-|a|^{2}\right) \leq \frac{6}{\min \{\eta, s\}} .
$$

(ii) For each $0<t<1$ there exists a constant $C=C(\eta, s, t)$ with $1<C<\infty$ such that

$$
C^{-1} \leq\left|\frac{w^{\prime}\left(z_{1}\right)}{w^{\prime}\left(z_{2}\right)}\right| \leq C, \quad z_{1}, z_{2} \in \Delta_{p}(a, t \cdot \min \{\eta, s\}) .
$$

Proof. (i) Let $\nu=\min \{\eta, s\}$. Since $g_{a}(z)=w\left(\varphi_{a}(\nu z)\right)$ in univalent and analytic in $\mathbb{D}$, we deduce

$$
\sup _{z \in \mathbb{D}}\left|\left(1-|z|^{2}\right) \frac{g_{a}^{\prime \prime}(z)}{g_{a}^{\prime}(z)}-2 \bar{z}\right| \leq 4
$$

by [15, Lemma 1.3]. Hence

$$
\left|\frac{w^{\prime \prime}(a)}{w^{\prime}(a)}\left(1-|a|^{2}\right) \nu-2 \bar{a} \nu\right|=\left|\frac{g_{a}^{\prime \prime}(0)}{g_{a}^{\prime}(0)}\right| \leq 4,
$$

from which the assertion follows.

(ii) As above, let $\nu=\min \{\eta, s\}$. Since $g_{a}(z)=w\left(\varphi_{a}(\nu z)\right)$ is analytic and univalent in $\mathbb{D}, \log g_{a}^{\prime}$ is a well-defined analytic function whose Bloch-norm satisfies $\left\|\log g_{a}^{\prime}\right\|_{\mathcal{B}} \leq 6$ by (9.3). For more information on Bloch functions we refer to [1]. Recall that Bloch functions are precisely those analytic functions in $\mathbb{D}$ which are Lipschitz when the unit disc is endowed with the hyperbolic metric and the plane 
with the Euclidean metric. Hence, if $\zeta_{1}, \zeta_{2} \in D(0, t)$ for some fixed $0<t<1$, then [8, Proposition 1, p. 43] implies

$$
|\log | \frac{g_{a}^{\prime}\left(\zeta_{1}\right)}{g_{a}^{\prime}\left(\zeta_{2}\right)}|| \leq \frac{\left\|\log g_{a}^{\prime}\right\|_{\mathcal{B}}}{2} \log \frac{1+\varrho_{p}\left(\zeta_{1}, \zeta_{2}\right)}{1-\varrho_{p}\left(\zeta_{1}, \zeta_{2}\right)} \leq 3 \log \frac{1+\frac{2 t}{1+t^{2}}}{1-\frac{2 t}{1+t^{2}}}
$$

Let $K=K(t)$ be the constant defined by the right-hand side of (9.4). Consequently,

$$
e^{-K} \leq\left|\frac{g_{a}^{\prime}\left(\zeta_{1}\right)}{g_{a}^{\prime}\left(\zeta_{2}\right)}\right| \leq e^{K}, \quad \zeta_{1}, \zeta_{2} \in D(0, t)
$$

from which (9.2) follows for $C=e^{K}(1+\nu)^{2} /(1-\nu)^{2}$.

For $n \in \mathbb{N}$, the arcs

$$
\left\{e^{i \theta} \in \partial \mathbb{D}:(j-1) 2^{-n+2} \pi \leq \theta \leq j 2^{-n+2} \pi\right\}, \quad j=1, \ldots, 2^{n-1},
$$

having pairwise disjoint interiors, constitute the $n^{\text {th }}$ generation of dyadic subintervals of $\partial \mathbb{D}$ - the first generation being $\partial \mathbb{D}$ itself. Analogously, we may define dyadic subintervals of any $\operatorname{arc} I \subset \partial \mathbb{D}$.

The set

$$
Q=Q_{I}=\{z \in \mathbb{D}: 1-|I| /(2 \pi) \leq|z|<1, \arg z \in I\}
$$

is called a Carleson square, where the interval $I \subset \partial \mathbb{D}$ is said to be the base of $Q$. The length of $Q$ is defined to be $\ell(Q)=|I|$ (the arc-length of $I$ ), while the top part (or the top half) of $Q$ is

$$
T(Q)=\{z \in Q: 1-\ell(Q) /(2 \pi) \leq|z| \leq 1-\ell(Q) /(4 \pi)\} .
$$

Let $z_{Q}$ denote the center point of $T(Q)$. Dyadic subsquares of a Carleson square $Q$ are those Carleson squares whose bases are dyadic subintervals of the base of $Q$. Finally, a Carleson square $S$ is said to the father of $Q$ provided that $Q$ is a dyadic subsquare of $S$ and $\ell(Q)=\ell(S) / 2$.

The following lemma is reminiscent of Lemma 11(ii), and hence its proof is omitted. The key point is that the top part of each Carleson square can be covered by finitely many pseudo-hyperbolic discs of fixed radius.

Lemma 12. Let $w \in \mathcal{U}(\eta)$ for some $0<\eta \leq 1$, and let $w^{\prime}$ be normal. Suppose that $s=s\left(\sigma\left(w^{\prime}\right)\right)$ is a constant such that (19.1) holds, and define $\lambda=(9 / 10+s) /(1+$ $9 s / 10)$. Then, there exists a constant $C_{0}=C_{0}\left(\eta, \sigma\left(w^{\prime}\right)\right)$ with $1<C_{0}<\infty$ such that the following conclusions hold.

(i) If $Q$ is a Carleson square such that $\varrho_{p}\left(T(Q), \mathcal{P}_{w}\right) \geq \lambda$, and $S$ is the fathersquare of $Q$, then

$$
C_{0}^{-1} \leq\left|\frac{w^{\prime}\left(z_{Q}\right)}{w^{\prime}\left(z_{S}\right)}\right| \leq C_{0}
$$

The same conclusion holds if $Q$ is the father-square of $S$.

(ii) If $Q$ is a Carleson square such that $\varrho_{p}\left(T(Q), \mathcal{P}_{w}\right) \geq \lambda$, then

$$
C_{0}^{-1} \leq\left|\frac{w^{\prime}\left(z_{1}\right)}{w^{\prime}\left(z_{2}\right)}\right| \leq C_{0}, \quad z_{1}, z_{2} \in T(Q) .
$$

(iii) If $Q$ is a Carleson square such that $\varrho_{p}\left(T(Q), \mathcal{P}_{w}\right)<\lambda$, then

$$
\left|w^{\prime}(z)\right| \geq C_{0}^{-1}, \quad z \in T(Q) .
$$


The next result, which is based on an argument similar to that of [3, Theorem 4], allows us to control the number of those Carleson squares where $w^{\prime}$ is small. This information is crucial for our purposes since we want to prove that $1 / w^{\prime}$ belongs to some Hardy space.

Lemma 13. Let $w$ be meromorphic in $\mathbb{D}$ such that $\left|S_{w}(z)\right|^{2}\left(1-|z|^{2}\right)^{3} d m(z)$ is a Carleson measure, and let $w^{\prime}$ be normal. Suppose that $C_{0}=C_{0}\left(\left\|S_{w}\right\|_{F^{2}}, \sigma\left(w^{\prime}\right)\right)$ with $1<C_{0}<\infty$ is the constant ensured by Lemma 12. Then, there exists a constant $\varepsilon_{0}=\varepsilon_{0}\left(\left\|S_{w}\right\|_{F^{2}}, \sigma\left(w^{\prime}\right)\right)$ with $0<\varepsilon_{0}<\min \left\{1 / 4, C_{0}^{-1}\right\}$ having the following property:

If $Q$ is a Carleson square satisfying $\left|w^{\prime}\left(z_{Q}\right)\right| \leq C_{0}^{-1 / \varepsilon_{0}}$, and $\left\{Q_{j}\right\}_{j=1}^{\infty}$ is the collection of maximal (with respect of inclusion) dyadic subsquares of $Q$ for which either

$$
\text { (i) }\left|w^{\prime}\left(z_{Q_{j}}\right)\right| \leq \varepsilon_{0}\left|w^{\prime}\left(z_{Q}\right)\right| \quad \text { or } \quad \text { (ii) }\left|w^{\prime}\left(z_{Q_{j}}\right)\right| \geq C_{0}^{-2},
$$

then $\sum_{j=1}^{\infty} \ell\left(Q_{j}\right) \leq \ell(Q) / 2$.

Proof. Let $\mathcal{R}=Q \backslash \bigcup_{j=1}^{\infty} Q_{j}$. By Lemma 12(iii), $\mathcal{R}$ is a simply connected subset of $\mathbb{D}$, which does not contain any poles of $w$ (nor poles of $w^{\prime}$ for that matter). Even more is true, the pseudo-hyperbolic neighborhood of the radius $\lambda=\lambda\left(\sigma\left(w^{\prime}\right)\right)$ of $\mathcal{R}$ does not contain any poles of $w$, see Lemma 12 for the precise definition of $\lambda$.

The function $\log w^{\prime}$ is analytic in $\mathcal{R}$. By a standard limiting argument we may assume that $\mathcal{R}$ is compactly contained in $\mathbb{D}$. We know that $\mathcal{R}$ is a chord-arc domain 1 [2, p. 25] with some absolute chord-arc constant $1 \leq C<\infty$. Let $\Phi(z)=$ $\left(z-z_{Q}\right) / \ell(Q)$, and denote $\mathcal{D}=\Phi(\mathcal{R})$. Now, $\mathcal{D}$ is a simply connected bounded chord-arc domain, which contains the origin. Since $d\left(z_{Q}, \partial \mathcal{R}\right) \asymp \operatorname{Diam}(\mathcal{R}) \asymp \ell(Q)$, we have $d(0, \partial \mathcal{D}) \asymp \operatorname{Diam}(\mathcal{D}) \asymp 1$, where $d$ denotes the Euclidean distance while Diam is the Euclidean diameter.

Define $F(\zeta)=\log w^{\prime}\left(\Phi^{-1}(\zeta)\right)-\log w^{\prime}\left(\Phi^{-1}(0)\right)$ for $\zeta \in \mathcal{D}$, and note that $F$ is analytic in $\mathcal{D}$. We have

$$
\int_{\mathcal{D}}\left|F^{\prime \prime}(\zeta)\right|^{2} d(\zeta, \partial \mathcal{D})^{3} d m(\zeta)=\frac{1}{\ell(Q)} \int_{\mathcal{R}}\left|\left(\log w^{\prime}\right)^{\prime \prime}(z)\right|^{2} d(z, \partial \mathcal{R})^{3} d m(z),
$$

where $z=\Phi^{-1}(\zeta)$ and $d(\zeta, \partial \mathcal{D})=d(z, \partial \mathcal{R}) / \ell(Q)$. By the identity $\left(\log w^{\prime}\right)^{\prime \prime}=$ $S_{w}+2^{-1}\left(w^{\prime \prime} / w^{\prime}\right)^{2}$, the estimate $d(z, \partial \mathcal{R}) \leq 1-|z|^{2}$ for all $z \in \mathcal{R}$, and the assumption that $\left|S_{w}(z)\right|^{2}\left(1-|z|^{2}\right)^{3} d m(z)$ is a Carleson measure, (9.6) implies

$$
\begin{aligned}
\int_{\mathcal{D}}\left|F^{\prime \prime}(\zeta)\right|^{2} d(\zeta, \partial \mathcal{D})^{3} d m(\zeta) \\
\quad \lesssim \frac{1}{\ell(Q)} \int_{\mathcal{R}}\left|S_{w}(z)\right|^{2}\left(1-|z|^{2}\right)^{3} d m(z)+\frac{1}{\ell(Q)} \int_{\mathcal{R}}\left|\frac{w^{\prime \prime}(z)}{w^{\prime}(z)}\right|^{4} d(z, \partial \mathcal{R})^{3} d m(z) \\
\quad \lesssim\left\|S_{w}\right\|_{F^{2}}^{2}+\frac{1}{\ell(Q)} \int_{\mathcal{R}}\left|\frac{w^{\prime \prime}(z)}{w^{\prime}(z)}\right|^{4} d(z, \partial \mathcal{R})^{3} d m(z)
\end{aligned}
$$

with absolute comparison constants.

\footnotetext{
${ }^{1}$ If $\gamma \subset \mathbb{C}$ is a locally rectifiable closed curve, and there exists a constant $1 \leq C<\infty$ such that the shorter arc connecting any two points $z_{1}, z_{2} \in \gamma$ has arc-length at most $C\left|z_{1}-z_{2}\right|$, then $\gamma$ is called chord-arc. In particular, a domain in $\mathbb{C}$ is called chord-arc if its boundary is a chord-arc curve. Chord-arc curves are also known as Lavrentiev curves, and they are precisely the bi-Lipschitz images of circles.
} 
Our argument is based on the following auxiliary result, whose proof is omitted. The proof of Lemma 14 is a laborious but straightforward modification of the argument in [3, pp. 105-107].

Lemma 14. Under the assumptions of Lemma 13: For each $0<\varepsilon<\infty$ there exists a constant $C_{1}=C_{1}\left(\varepsilon,\left\|S_{w}\right\|_{F^{2}}, \sigma\left(w^{\prime}\right)\right)$ with $0<C_{1}<\infty$ such that

$$
\int_{\mathcal{R}}\left|\frac{w^{\prime \prime}(z)}{w^{\prime}(z)}\right|^{4} d(z, \partial \mathcal{R})^{3} d m(z) \leq C_{1} \ell(Q)+\varepsilon^{2} \int_{\mathcal{R}}\left|\frac{w^{\prime \prime}(z)}{w^{\prime}(z)}\right|^{2} d(z, \partial \mathcal{R}) d m(z) .
$$

We continue with the proof of Lemma 13, By combining (9.7) and (9.8), the change of variable gives

$$
\int_{\mathcal{D}}\left|F^{\prime \prime}(\zeta)\right|^{2} d(\zeta, \partial \mathcal{D})^{3} d m(\zeta) \lesssim\left\|S_{w}\right\|_{F^{2}}^{2}+C_{1}+\varepsilon^{2} \int_{\mathcal{D}}\left|F^{\prime}(\zeta)\right|^{2} d(\zeta, \partial \mathcal{D}) d m(\zeta)
$$

with absolute comparison constants. We apply a well-known version of Green's formula [3, Lemma 3.6] for the domain $\mathcal{D}$. Since $F(0)=0$ and $\ell(Q) \leq(4 / 3)(1-$ $\left|z_{Q}\right|^{2}$ ), Lemma 11(i) and (9.9) imply

$$
\begin{aligned}
\int_{\partial \mathcal{D}}|F(\zeta)|^{2}|d \zeta| & \lesssim\left|F^{\prime}(0)\right|^{2}+\int_{\mathcal{D}}\left|F^{\prime \prime}(\zeta)\right|^{2} d(\zeta, \partial \mathcal{D})^{3} d m(\zeta) \\
& \lesssim\left|\frac{w^{\prime \prime}\left(z_{Q}\right)}{w^{\prime}\left(z_{Q}\right)}\right|^{2} \ell(Q)^{2}+\left\|S_{w}\right\|_{F^{2}}^{2}+C_{1}+\varepsilon^{2} \int_{\mathcal{D}}\left|F^{\prime}(\zeta)\right|^{2} d(\zeta, \partial \mathcal{D}) d m(\zeta) \\
& \lesssim\left(\frac{1}{\min \{\eta, s\}}\right)^{2}+\left\|S_{w}\right\|_{F^{2}}^{2}+C_{1}+\varepsilon^{2} \int_{\partial \mathcal{D}}|F(\zeta)|^{2}|d \zeta|
\end{aligned}
$$

with absolute comparison constants. If $0<\varepsilon<\infty$ is sufficiently small, then the computation above shows that there exists a constant $C_{2}=C_{2}\left(\left\|S_{w}\right\|_{F^{2}}, \sigma\left(w^{\prime}\right)\right)$ with $0<C_{2}<\infty$ such that

$$
\int_{\partial \mathcal{R}}\left|\log w^{\prime}(z)-\log w^{\prime}\left(z_{Q}\right)\right|^{2}|d z|=\ell(Q) \int_{\partial \mathcal{D}}|F(\zeta)|^{2}|d \zeta| \leq C_{2} \ell(Q) .
$$

Let $T_{j}$ denote the top of $\partial Q_{j}$ (i.e. the roof of $Q_{j}$ ) for $j \in \mathbb{N}$. Since the roofs $T_{j} \subset \partial \mathcal{R}$ are pairwise disjoint, we deduce from (9.10) that

$$
\sum_{j=1}^{\infty} \int_{T_{j}}\left|\log w^{\prime}(z)-\log w^{\prime}\left(z_{Q}\right)\right|^{2}|d z| \leq C_{2} \ell(Q) .
$$

There are two types of subsquares $Q_{j}$, which result from (9.5).

(i) In the case of type (i) squares, Lemma 12(iii) shows that $\varrho_{p}\left(T\left(Q_{j}\right), \mathcal{P}_{w}\right) \geq \lambda$. Hence Lemma 12(ii) implies

$$
\left|w^{\prime}(z)\right| \leq C_{0}\left|w^{\prime}\left(z_{Q_{j}}\right)\right| \leq C_{0} \varepsilon_{0}\left|w^{\prime}\left(z_{Q}\right)\right|, \quad z \in T_{j},
$$

and further,

$$
\begin{aligned}
\left|\log w^{\prime}(z)-\log w^{\prime}\left(z_{Q}\right)\right| & \geq \log \left|w^{\prime}\left(z_{Q}\right)\right|-\log \left|w^{\prime}(z)\right| \\
& \geq \log \left(C_{0} \varepsilon_{0}\right)^{-1}>0, \quad z \in T_{j} .
\end{aligned}
$$

(ii) In the case of type (ii) squares $Q_{j}$, let $S_{j}$ be their father-squares, respectively. Now, by Lemma 12

$$
\left|w^{\prime}(z)\right| \geq C_{0}^{-1}\left|w^{\prime}\left(z_{S_{j}}\right)\right| \geq C_{0}^{-2}\left|w^{\prime}\left(z_{Q_{j}}\right)\right| \geq C_{0}^{-4}, \quad z \in T_{j} .
$$


Since $\left|w^{\prime}\left(z_{Q}\right)\right| \leq C_{0}^{-1 / \varepsilon_{0}}$, and $\varepsilon_{0}<1 / 4$, we get

$$
\begin{aligned}
\left|\log w^{\prime}(z)-\log w^{\prime}\left(z_{Q}\right)\right| & \geq \log \left|w^{\prime}(z)\right|-\log \left|w^{\prime}\left(z_{Q}\right)\right| \\
& =\left(\varepsilon_{0}^{-1}-4\right) \log C_{0}>0, \quad z \in T_{j} .
\end{aligned}
$$

By combining (9.11), (9.12) and (9.13), and by choosing $\varepsilon_{0}=\varepsilon_{0}\left(\left\|S_{w}\right\|_{F^{2}}, \sigma\left(w^{\prime}\right)\right)$ with $0<\varepsilon_{0}<C_{0}^{-1}$ sufficiently small, we conclude $\sum_{j=1}^{\infty} \ell\left(Q_{j}\right) \leq 2^{-1} \ell(Q)$. The assertion of Lemma 13 follows.

\section{Proof of Proposition 7}

Let $f$ be a solution of (1.1) with $A \in H_{2}^{\infty}$, and let $\left\{z_{n}\right\}_{n=1}^{\infty}$ be the zero-sequence of $f$. Implication (i) $\Rightarrow$ (ii) is a direct consequence of (2.5), and hence it suffices to prove (ii) $\Rightarrow$ (iii) and (iii) $\Rightarrow$ (i).

(ii) $\Rightarrow$ (iii): Denote $K=\sup _{n \in \mathbb{N}}\left(1-\left|z_{n}\right|^{2}\right)\left|f^{\prime}\left(z_{n}\right)\right|<\infty$. Fix $n \in \mathbb{N}$, and let $z \in \bar{D}\left(z_{n}, c\left(1-\left|z_{n}\right|\right)\right)$ be any point satisfying

$$
\max _{\zeta \in \bar{D}\left(z_{n}, c\left(1-\left|z_{n}\right|\right)\right)}|f(\zeta)|=|f(z)| .
$$

Here $\bar{D}\left(z_{n}, c\left(1-\left|z_{n}\right|\right)\right)$ denotes a closed Euclidean disc centered at $z_{n} \in \mathbb{D}$, and $c$ is a sufficiently small constant (to be defined later). By means of (1.1) we deduce

$$
\begin{aligned}
|f(z)| & =\left|\int_{z_{n}}^{z}\left(f^{\prime}\left(z_{n}\right)+\int_{z_{n}}^{\zeta} f^{\prime \prime}(s) d s\right) d \zeta\right| \\
& \leq c\left(1-\left|z_{n}\right|\right)\left|f^{\prime}\left(z_{n}\right)\right|+|f(z)| \int_{z_{n}}^{z} \int_{z_{n}}^{\zeta} \frac{\|A\|_{H_{2}^{\infty}}}{(1-|s|)^{2}}|d s||d \zeta| \\
& \leq c K+|f(z)| \frac{c^{2}\left(1-\left|z_{n}\right|\right)^{2}\|A\|_{H_{2}^{\infty}}}{(1-c)^{2}\left(1-\left|z_{n}\right|\right)^{2}} .
\end{aligned}
$$

If $c=c\left(\|A\|_{H_{2}^{\infty}}\right)$ is sufficiently small to satisfy $c^{2}\|A\|_{H_{2}^{\infty}} /(1-c)^{2}<1$, then we get

$$
|f(\zeta)| \leq \frac{c K}{1-c^{2}\|A\|_{H_{2}^{\infty}} /(1-c)^{2}}, \quad \zeta \in \bigcup_{n=1}^{\infty} D\left(z_{n}, c\left(1-\left|z_{n}\right|\right)\right) .
$$

(iii) $\Rightarrow($ i): Suppose that $f$ satisfies

$$
|f(z)| \leq C, \quad z \in \bigcup_{n=1}^{\infty} D\left(z_{n}, c\left(1-\left|z_{n}\right|\right)\right)
$$

for some constants $0<c<1$ and $0<C<\infty$. Let $g$ be a linearly independent solution to $f$ such that the Wronskian determinant $W(f, g)=1$. Define $w=g / f$, and notice that $S_{w}=2 A$ and $w^{\prime}=1 / f^{2}$. Now $S_{w} \in H_{2}^{\infty}$ implies $w \in \mathcal{U}(\eta)$ for any sufficiently small $\eta=\eta\left(\left\|S_{w}\right\|_{H_{2}^{\infty}}\right)$ by Nehari's theorem [16, Corollary 6.4]. Let $t=t\left(\left\|S_{w}\right\|_{H_{2}^{\infty}}, c\right)$ with $0<t<1$ be a sufficiently small constant such that

(a) $\bar{\Delta}_{p}\left(z_{n}, \eta t\right) \subset D\left(z_{n}, c\left(1-\left|z_{n}\right|\right)\right)$ for all $n \in \mathbb{N}$;

(b) $2 \eta t /\left(1+\eta^{2} t^{2}\right)<\eta$.

Here $\bar{\Delta}_{p}(a, \eta t)$ stands for the closed pseudo-hyperbolic disc of radius $\eta t$, centered at $a \in \mathbb{D}$. We proceed to verify (2.5) in two parts.

First, suppose that $a \in \mathbb{D}$ is a point such that $\varrho_{p}\left(a, \mathcal{P}_{w}\right) \geq \eta t$. By Lemma 11(i) we deduce

$$
\left(1-|a|^{2}\right) \frac{\left|f^{\prime}(a)\right|}{1+|f(a)|^{2}} \leq \frac{1}{4}\left(1-|a|^{2}\right) \frac{\left|w^{\prime \prime}(a)\right|}{\left|w^{\prime}(a)\right|} \leq \frac{3}{2 \eta t} .
$$


Second, suppose that $a \in \mathbb{D}$ is a point such that $\varrho_{p}\left(a, \mathcal{P}_{w}\right)<\eta t$, or equivalently, $a \in \Delta_{p}\left(z_{n}, \eta t\right)$ for some $n \in \mathbb{N}$. By the maximum modulus principle there exists a point $s_{n} \in \partial \Delta_{p}\left(z_{n}, \eta t\right)$ such that

$$
\max _{z \in \bar{\Delta}_{p}\left(z_{n}, \eta t\right)}\left|f^{\prime}(z)\right|=\left|f^{\prime}\left(s_{n}\right)\right| .
$$

Note that $\Delta_{p}\left(s_{n}, \eta t\right)$ does not contain any zeros of $f$ (any such zero would lie too close to $z_{n}$ by the condition (b) and the fact $\left.w \in \mathcal{U}(\eta)\right)$. Lemma 11(i) yields

$$
\left(1-\left|s_{n}\right|^{2}\right)\left|f^{\prime}\left(s_{n}\right)\right| \leq \frac{3}{\eta t}\left|f\left(s_{n}\right)\right| \text {. }
$$

Since $a, s_{n} \in \bar{\Delta}_{p}\left(z_{n}, \eta t\right)$, there exists a constant $K=K\left(\left\|S_{w}\right\|_{H_{2}^{\infty}}, c\right)$ with $1<K<$ $\infty$ such that $1 / K \leq\left(1-|a|^{2}\right) /\left(1-\left|s_{n}\right|^{2}\right) \leq K$. By means of the maximum modulus principle, (10.1) and (10.2) we deduce

$$
\left(1-|a|^{2}\right) \frac{\left|f^{\prime}(a)\right|}{1+|f(a)|^{2}} \leq \frac{1-|a|^{2}}{1-\left|s_{n}\right|^{2}}\left(\left(1-\left|s_{n}\right|^{2}\right)\left|f^{\prime}\left(s_{n}\right)\right|\right) \frac{1}{1+|f(a)|^{2}} \leq \frac{3 C K}{\eta t} .
$$

We have proved $\sigma(f)<\infty$, and hence $f$ is normal. This concludes the proof of Proposition 7.

\section{Proof of Theorem 8}

We proceed to show that the non-tangential maximal function

$$
\left(1 / w^{\prime}\right)^{\star}\left(e^{i \theta}\right)=\sup _{z \in \Gamma_{\alpha}\left(e^{i \theta}\right)} \frac{1}{\left|w^{\prime}(z)\right|}, \quad e^{i \theta} \in \partial \mathbb{D},
$$

belongs to the weak Lebesgue space $L_{w}^{p}(\partial \mathbb{D})$ for some $0<p<\infty$, which is to say that there exists a constant $C=C\left(\alpha, w^{\prime}\right)$ with $0<C<\infty$ such that the distribution function satisfies

$$
\left|\left\{e^{i \theta} \in \partial \mathbb{D}:\left(1 / w^{\prime}\right)^{\star}\left(e^{i \theta}\right)>\lambda\right\}\right| \leq \frac{C}{\lambda^{p}}, \quad 0<\lambda<\infty .
$$

This leads to the assertion, since $L_{w}^{p}(\partial \mathbb{D}) \subset L^{q}(\partial \mathbb{D})$ for any $0<q<p$. Here $\Gamma_{\alpha}\left(e^{i \theta}\right)=\left\{z \in \mathbb{D}:\left|z-e^{i \theta}\right| \leq \alpha(1-|z|)\right\}$, for fixed $1<\alpha<\infty$, is a non-tangential approach region with vertex at $e^{i \theta} \in \partial \mathbb{D}$ with aperture of $2 \arctan \sqrt{\alpha^{2}-1}$, and the absolute value of the set is its one dimensional Lebesgue measure.

Let $C_{0}=C_{0}\left(\left\|S_{w}\right\|_{F^{2}}, \sigma\left(w^{\prime}\right)\right)$ with $1<C_{0}<\infty$ be the constant ensured by Lemma 12, and $\varepsilon_{0}=\varepsilon_{0}\left(\left\|S_{w}\right\|_{F^{2}}, \sigma\left(w^{\prime}\right)\right)$ with $0<\varepsilon_{0}<\min \left\{1 / 4, C_{0}^{-1}\right\}$ be the constant resulting from Lemma 13. We consider the collection $\mathcal{G}_{0}$ of maximal (with respect to inclusion) dyadic subsquares of $\mathbb{D}$, of at least second generation, satisfying

$$
\left|w^{\prime}\left(z_{Q}\right)\right| \leq C_{0}^{-1 / \varepsilon_{0}}, \quad Q \in \mathcal{G}_{0} .
$$

Denote $L=L\left(C_{0}, \varepsilon_{0}\right)=C_{0}^{1+1 / \varepsilon_{0}}$ and $M=M\left(C_{0}, \varepsilon_{0}\right)=C_{0} / \varepsilon_{0}$, for short. The maximality and Lemma 12 imply

$$
\left|w^{\prime}(z)\right|>L^{-1}, \quad z \in \mathbb{D} \backslash \bigcup_{Q \in \mathcal{G}_{0}} Q
$$

and $\left|w^{\prime}\left(z_{Q}\right)\right|>L^{-1}$ for all $Q \in \mathcal{G}_{0}$.

Apply Lemma 13 to each $Q \in \mathcal{G}_{0}$ to get the collection $\mathcal{S}_{Q}$ of dyadic subsquares of $Q$, where the subsquares are maximal with the property

$$
\left|w^{\prime}\left(z_{S}\right)\right| \leq \varepsilon_{0}\left|w^{\prime}\left(z_{Q}\right)\right|, \quad S \in \mathcal{S}_{Q} .
$$


Note that the squares in the family $\mathcal{S}_{Q}$ are either the ones appearing in (i) of Lemma 13 or are contained in the ones appearing in (ii). Let $\mathcal{G}_{1}=\bigcup_{Q \in \mathcal{G}_{0}} \mathcal{S}_{Q}$. For any $Q \in \mathcal{G}_{0}$, the maximality and Lemma 12 yield

$$
\left|w^{\prime}(z)\right|>\varepsilon_{0}\left|w^{\prime}\left(z_{Q}\right)\right| C_{0}^{-1}>L^{-1} M^{-1}, \quad z \in \mathbb{D} \backslash \bigcup_{Q^{\star} \in \mathcal{G}_{1}} Q^{\star}
$$

$\left|w^{\prime}\left(z_{S}\right)\right|>\varepsilon_{0}\left|w^{\prime}\left(z_{Q}\right)\right| C_{0}^{-1}>L^{-1} M^{-1}$ for all $S \in \mathcal{S}_{Q}$; and moreover, we have $\sum_{S \in \mathcal{S}_{Q}} \ell(S) \leq \ell(Q) / 2$ by Lemma 13. We deduce

$$
\sum_{Q \in \mathcal{G}_{1}} \ell(Q) \leq \frac{1}{2} \sum_{Q \in \mathcal{G}_{0}} \ell(Q)
$$

Repeating this process inductively, we obtain collections $\mathcal{G}_{n}$ for $n \in \mathbb{N}$ such that

$$
\left|w^{\prime}(z)\right|>L^{-1} M^{-n}, \quad z \in \mathbb{D} \backslash \bigcup_{Q \in \mathcal{G}_{n}}^{\infty} Q, \quad n \in \mathbb{N},
$$

and

$$
\sum_{Q \in \mathcal{G}_{n}} \ell(Q) \leq \frac{1}{2^{n}} \sum_{Q \in \mathcal{G}_{0}} \ell(Q), \quad n \in \mathbb{N}
$$

Fix any $\lambda$ for which $L M \leq \lambda<\infty$, and choose the natural number $N$ such that $L M^{N} \leq \lambda<L M^{N+1}$. Now

$$
\begin{aligned}
\left\{e^{i \theta} \in \partial \mathbb{D}:\left(1 / w^{\prime}\right)^{\star}\left(e^{i \theta}\right)>\lambda\right\} & =\left\{e^{i \theta} \in \partial \mathbb{D}: \inf _{z \in \Gamma_{\alpha}\left(e^{i \theta}\right)}\left|w^{\prime}(z)\right|<1 / \lambda\right\} \\
& \subset\left\{e^{i \theta} \in \partial \mathbb{D}: \inf _{z \in \Gamma_{\alpha}\left(e^{i \theta}\right)}\left|w^{\prime}(z)\right|<L^{-1} M^{-N}\right\} .
\end{aligned}
$$

By the inductive process above, there exists a constant $K=K(\alpha)$ with $1<K<\infty$ such that

$$
\left|\left\{e^{i \theta} \in \partial \mathbb{D}:\left(1 / w^{\prime}\right)^{\star}\left(e^{i \theta}\right)>\lambda\right\}\right| \leq \frac{K}{2^{N}} \sum_{Q \in \mathcal{G}_{0}} \ell(Q) \leq \frac{4 \pi K L^{\left(\log _{2} M\right)^{-1}}}{\lambda^{\left(\log _{2} M\right)^{-1}}} .
$$

Hence $\left(1 / w^{\prime}\right)^{\star} \in L_{w}^{p}(\partial \mathbb{D})$ for $p=1 /\left(\log _{2} C_{0} / \varepsilon_{0}\right)$, and consequently, $1 / w^{\prime} \in H^{q}$ for any $0<q<p$. This proves the assertion of Theorem 8 .

\section{Proof of Corollary 9}

Let $f$ be a normal non-trivial solution of (1.1), where $|A(z)|^{2}\left(1-|z|^{2}\right)^{3} d m(z)$ is a Carleson measure. Let $g$ be a solution of (1.1) such that $W(f, g)=1$. Now $w=g / f$ satisfies $S_{w}=2 A$. Since $w^{\prime}=1 / f^{2}$ is normal $\left(\sigma\left(w^{\prime}\right) \leq 2 \sigma(f)<\infty\right)$, Theorem 8 asserts that for any sufficiently small $0<p<\infty$ there exists a constant $C=C\left(p,\|A\|_{F^{2}}, \sigma(f)\right)$ with $1<C<\infty$ such that $\|f\|_{H^{2 p}}^{2 p}=\left\|1 / w^{\prime}\right\|_{H^{p}}^{p} \leq C$.

\section{REFERENCES}

[1] J.M. Anderson, J. Clunie and Ch. Pommerenke, On Bloch functions and normal functions, J. Reine Angew. Math. 270 (1974), 12-37.

[2] C. Bishop, Non-rectifiable limit sets of dimension one, Rev. Mat. Iberoamericana 18 (2002), no. 3, 653-684.

[3] C. Bishop, P. Jones, Harmonic measure, $L^{2}$ estimates and the Schwarzian derivative, J. Anal. Math. 62 (1994), 77-113.

[4] M. Chuaqui, J. Gröhn, J. Heittokangas, J. Rättyä, Zero separation results for solutions of second order linear differential equations, Adv. Math. 245 (2013), 382-422. 
[5] J.A. Cima, G. Schober, Analytic functions with bounded mean oscillation and logarithms of $H^{p}$ functions, Math. Z. 151 (1976), no. 3, 295-300.

[6] J. Conway, Functions of One Complex Variable II, Graduate Texts in Mathematics, 159. Springer-Verlag, New York, 1995.

[7] P. Duren, Theory of $H^{p}$ Spaces, Dover, New York, 2000.

[8] P. Duren, A. Schuster, Bergman Spaces, Amer. Math. Soc., Providence, RI, 2004.

[9] J. Garnett, Bounded Analytic Functions, revised 1st ed., Springer, New York, 2007.

[10] J. Heittokangas, On complex differential equations in the unit disc, Ann. Acad. Sci. Fenn. Math. Diss. No. 122 (2000), 54 pp.

[11] J. Heittokangas, A survey of Blaschke-oscillatory differential equations, with updates, Fields Inst. Commun., 65, Springer, New York, 2013.

[12] E.L. Ince, Ordinary Differential Equations, Dover Publications, New York, 1944.

[13] O. Lehto, K.I. Virtanen, Boundary behaviour and normal meromorphic functions, Acta Math. 97 (1957), 47-65.

[14] Z. Nehari, The Schwarzian derivative and schlicht functions, Bull. Amer. Math. Soc. 55 (1949), 545-551.

[15] Ch. Pommerenke, Univalent Functions, Vandenhoeck \& Ruprecht, Göttingen, 1975.

[16] Ch. Pommerenke, On the mean growth of the solutions of complex linear differential equations in the disk, Complex Variables Theory Appl. 1 (1982/83), no. 1, 23-38.

[17] J. Rättyä, Linear differential equations with solutions in Hardy spaces, Complex Var. Elliptic Equ. 52 (2007), no. 9, 785-795.

[18] B. Schwarz, Complex nonoscillation theorems and criteria of univalence, Trans. Amer. Math. Soc. 80 (1955), 159-186.

[19] S. Yamashita, Schlicht holomorphic functions and the Riccati differential equation, Math. Z. 157 (1977), no. 1, 19-22.

[20] S. Yamashita, Area integrals for normal and Yosida functions, Proc. Japan Acad. Ser. A Math. Sci. 62 (1986), no. 5, 185-188.

Department of Physics and Mathematics, University of Eastern Finland, P.O. Box 111, FI-80101 JoEnsuU, Finland

E-mail address: janne.grohn@uef.fi

Departament de Matemàtiques, Universitat Autònoma de Barcelona, 08193, BelLATERra, BARCELONA, Spain

E-mail address: artur@mat.uab.cat

Department of Physics and Mathematics, University of Eastern Finland, P.O. Box 111, FI-80101 JoensuU, Finland

E-mail address: jouni.rattya@uef.fi 